\title{
Commonly Deployed-Employed African Neonatal Skin Care Products Compromise Epidermal Function in Mice
}

Mao-Qiang Man, M.D. ${ }^{1}$, Richard Sun, B.A. ${ }^{1}$, George Man ${ }^{1}$, Dale Lee ${ }^{1}$, Zelee Hill, Ph.D. ${ }^{2}$, Peter M. Elias, M.D. ${ }^{1}$

${ }^{1}$ Dermatology Service, Veterans Affairs Medical Center, and Department of Dermatology, University of California San Francisco, CA, USA; ${ }^{2}$ Institute for Global Health, Faculty of Population Health Sciences, University College London, London, the United Kingdom.

Address Correspondence To:

Mao-Qiang Man, MD

Dermatology (190)

4150 Clement Street

San Francisco, CA 94121

USA

Tel: (415)750-2091

Fax: (415)750-2106

E Mail: mqman@hotmail.com

Key Words: Epidermal Permeability Barrier, Emollient, Stratum Corneum Hydration, Skin pH

Running Title: Topical African Emollients Perturb Epidermal Function

Abbreviations: TEWL, Transepidermal Water Loss; SC, Stratum Corneum.

All authors declare no conflicts of interest.

All animal procedures were approved by the Animal Studies Subcommittee (IACUC) of the San Francisco Veterans Administration Medical Center and performed in accordance with their guidelines. 


\begin{abstract}
Background: Neonatal mortality rates are much higher in the developing world than in developed countries. Infections are a major cause for neonatal death, particularly in preterm infants where a defective epidermal permeability barrier function facilitates transcutaneous pathogen invasion. Objectives: To determine whether neonatal skin care products, commonly used in Africa, benefit or compromise epidermal functions in murine skin. Methods: Following the twice daily treatment of 6-8 weeks old hairless mice with each skin care product for 3 days, epidermal permeability barrier function, skin surface $\mathrm{pH}$, stratum corneum hydration and barrier recovery were measured with an MPA5 physiology monitor. For products showing some benefits in these initial tests, the epidermal permeability barrier homeostasis was assessed 1 and 5 hours after a single application to acutely disrupted skin. Results: All of the skin care products compromised both basal permeability barrier function and barrier repair kinetics. Moreover, after 3-day treatments, most of the products also reduced stratum corneum hydration, while also elevating skin surface $\mathrm{pH}$ to abnormal levels. Conclusions: These results demonstrate that some neonatal skin care products that are widely used in Africa perturb key epidermal functions, including permeability barrier homeostasis in mice. Should these products display similar effects on newborn human skin, they could cause a defective epidermal permeability barrier, which can increase body fluid loss, impair thermoregulation, and contribute to the high rates of neonatal morbidity and mortality that occur in Africa. Accordingly, alternative products that enhance permeability barrier function should be identified, particularly for use in preterm infants.
\end{abstract}




\section{Introduction}

Mortality rates are declining year by year worldwide [1], but neonatal mortality rates still exceed $4 \%$ in the developing world, accounting for $44 \%$ of all under-5 deaths [2]. Among contributors to neonatal mortality, prematurity, low birth_weight, and perinatal infections are the leading causes[3,4]. Mortality rates for pre-term ( $<37$ weeks gestational age) and low birth weight infants $(<2500 \mathrm{~g})$ are higher than for their full-_term and normal-normal-birth $\underline{\text { birth-weight }}$ counterparts [5-7]. Sixty to $80 \%$ of all neonatal deaths link to low birthweights [8], and preterm birth-associated complications are the leading cause of neonatal deaths in world -wide [9]. Preterm birth rates are particular high in Africa and Southeast Asia, ranging from $15-18 \%$, and the overall neonatal mortality rate is as high as $4.7 \%[5,10]$. Given the magnitude of the problem, the need to identify approaches that reduce neonatal mortality rates in the developing world, especially for pre-term and low birth weight infants is well recognized [11].

Skin plays an important role in preventing infections [12], but even full-term neonatal skin is not fully functional, which may increase the risk of infections and inflammatory dermatosees. Fullterm neonatal epidermis exhibits elevated transepidermal water loss (TEWL) rates, reduced

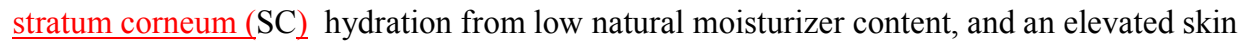
surface $\mathrm{pH}$ [13-15]. These abnormalities are further intensified in premature infants, who have a TEWL over 10-fold higher than their full-term counterparts, and they require a longer time to develop a mature permeability barrier than full-term infants [16-21].

Immature epidermal function in preterm infants may contribute to neonatal morbidity and mortality in several ways. First, a compromised permeability barrier and an elevated skin surface $\mathrm{pH}$ promote colonization by Staphylococcus aureus and other microbial pathogens ${ }_{2}$ in addition to 
an increase in bacterial invasion, even by non-pathogenic 'normal' flora [22-24]. Second, a defective permeability barrier can allow excessive fluid loss and cause an imbalance of serum electrolytes $[15,25]$. Third, elevated TEWL rates are associated with higher rates of energy consumption and loss of body weight [26,27]. Previous trials have shown that topical applications of permeability barrier enhancing emollients can reduce neonatal mortality among preterm infants by decreasing infection rates and increasing body weights [28-32]. Despite these promising results, the range of emollients that are applied to babies in the home is extensive, but we know little about which of these products enhance barrier function. The trials show a mortality reduction when either sunflower oil, Aquaphor or coconut oil were applied [28], but there is evidence from mouse models that mustard oil, commonly used to massage neonates across South East Asia, may be harmful to the skin [33]. In Africa, emollient use in newborns is very common, with a wide range of emollients used, including cow butter, shea butter, coconut oil as well as commercial baby oils and lotions [34]. Given their wide use, whether these emollients provide important benefits, or conversely whether they are potentially harmful is not

known. In this study, we assessed the impact of several widely used-emollients widely used in three African countries on epidermal function in mousee models.

\section{Materials and Methods}

Materials: Previous studies have demonstrated that albino hairless (Skh1) mice represent a suitable animal model for assessing the potential impact of skin care products on cutaneous structure and function in humans. For example, mouse epidermis comprises three cell layers [35], while preterm neonatal human epidermis displays a comparable number of nucleated cell layers [36]. Moreover, several studies have shown that topical products that enhance barrier function in murine skin, predict parallel results in normal human skin [e.g., 37]. Therefore, we used the 
widely-accepted hairless mouse model in the present study. Six-to-eight-week-old $6-8$ weeks old female SKH1 mice were purchased from Charles River Laboratories (Wilmington, MA). The emollients tested came from four African sites that were part of a pilot research study on local emollient use in Ekiti State in South West Nigeria, Borno State in North East Nigeria, the Oromia region of Ethiopia, and the Lindi and Mtwara regions of Tanzania. The sites and the formative research are described elsewhere [33]. Emollients were selected for testing because they are commonly used in the sites, but were of unknown benefit or harm. The exact ingredients in all products were unknown. The emollients tested included both manufactured and homemade butters, oils and lotions. Manufactured emollients were collected for testing from an unopened bottle purchased from a reputable shop in or close to the study sites, and homemade emollients were collected and stored as soon after production as possible.

Samples were collected in clear glass bottles that were filled to the top to reduce the potential for oxidation. Immediately after collection, s-amples were stored in the refrigerator and airfreighted with ice packs in insulated boxes to the University of California, San Francisco for analysis as described in the methods section below. If a similar emollient was commonly used in more than one site, then samples were taken from both sites as chemical composition can vary. In total two emollients were tested from Ethiopia (E): locally manufactured Vaseline ${ }^{\mathrm{TM}}$-(a-Unilever trademark $^{\mathrm{tm}}$ ofbrand petroleum jelly) and cow butter. Five from Borno State, Nigeria (B) were tested: shea butter, baby oil, olive oil , groundnut ôil and neem oil . Three from Ekiti State, Nigeria (EK) were tested: shea butter, baby lotion and olive oil. Five products from Lindi and Mtwara districts in Tanzania (T) were tested: Two types of cooking oil, two types of coconut oil and baby oil. Emollients were made between September, 2010 and January, 2012, and collected by the fieldworkers between December, 2011 and February, 2012, and analyzed between
Formatted: Font: (Default) Times New Roman, 12 pt

Formatted: Font: $16 \mathrm{pt}$ 
February and May, 2012.

Experimental protocols and functional studies: All animal procedures were approved by the

Formatted: Line spacing: Double Animal Studies Subcommittee (IACUC) of the San Francisco Veterans Administration Medical Center and performed in accordance with their guidelines. Experiments were carried out in a temperature and humidity controlled room. The temperature was $70 \pm 2^{\theta} \mathrm{F} \approx$ approximately $21 \pm 1^{0} \mathrm{C}$ and humidity ranged from $46 \%$ to $48 \%$. Both flanks of 10 mice were topically treated with individual emollients twice daily for three days. Butters were warmed to $45{ }^{0} \mathrm{C}$ before application; all other emollients were deployed at room temperature. Two to three products were tested alongside a control group of 10 untreated mice on each day.

Two to three products were tested on each day, and in the meanwhile, an additional group of Uㅡtreated mice served as normal controls.Epidermal functions were assessed as described previously $[38,39]$. Briefly, basal epidermal biophysical properties were assessed by measuring transepidermal water loss (TEWL) using a TM300 connected to MPA5 (C\&K, Cologne, Germany). For barrier recovery, changes in TEWL levels were measured at immediately after $(0$ $\underline{\text { time point) and three hours after tape stripping, which resulted in an } \approx \text { approximately } 10 \text {-fold }}$ increase in TEWL, and percent barrier recovery rates were calculated. The results were normalized to levels in untreated controls, setting the untreated controls as $100 \%$ For products showing some potential benefits in the initial tests or in previous studies, the effects of single application of individual emollients on barrier homeostasis in barrier-damaged mouse skin was determined. Products were applied topically immediately after barrier disruption in normal hairless mice. Untreated tape-stripped mice served as controls. TEWL was measured at 0,1 and 5 hours after tape stripping. 
Statistical Analyses: Data are expressed as mean \pm SEM. One-way ANOVA with Dunnett's Multiple Comparison Test was used to determine significant differences between treated and untreated groups.

\section{Results}

\section{Impact of African Emollients on Basal Barrier Function and Permeability Barrier}

\section{Homeostasis}

After three days of topical applications of each emollient to the flanks of separate cohorts of mice, no differences were observed in the overall macroscopic appearance of mouse skin, activity, or body weights between treated and untreated mice. As seen in Fig 1a, following 3 days of applications, most of the products significantly increased basal TEWL levels with the exception of baby lotion (EK) and olive oil (EK). Vaseline ${ }^{\mathrm{TM}}$ and cow butter (E), neem oil (B), coconut oil and baby oil (T) all provoked an over 3-fold increase in basal TEWL levels. Yet, supposedly similar products from different sites provoked variable responses. For example, olive oil from Borno (B) provoked a nearly 2--fold increase in basal TEWL, but olive oil (EK) did not change basal TEWL. These results demonstrate first, that most of the skin care products tested here disturb the basal permeability barrier;, and second, that the impact of supposedly comparable skin care products can vary with their sources.

Because barrier recovery kinetics is a more accurate predictor of cutaneous function, we next determined whether these products impact permeability barrier repair kinetics following 3 days of treatments. As seen in Fig 1b, most of the products delayed barrier recovery in comparison to untreated controls. Ground nut oil (B), olive oil (EK) and both coconut oils (T) had a particularly negative impact. Yet, certain products, including Vaseline ${ }^{\mathrm{TM}}-(\mathrm{E})$, shea butter (B and EK), and 
both cooking oils (T), while dramatically increasing basal TEWL (Fig 1a), did not impair barrier recovery kinetics in comparison to untreated controls (Fig 1b). Olive oil (EK), while causing no dramatic changes in basal TEWL, significantly delayed barrier recovery in comparison with untreated skin. Notably, baby lotion (EK) altered neither basal barrier function nor barrier repair kinetics. These results indicate that certain skin care products used in Africa diversely affect basal barrier and barrier repair kinetics.

Because previous studies showed that topical Vaseline ${ }^{\frac{\mathrm{TM}}{}}$ improves barrier function in acutely disrupted murine skin [40,41], and because we found that baby lotion (EK) did not significantly affect either basal TEWL or barrier repair kinetics, we next assessed the influence of these products on barrier function in barrier damaged skin. As seen in Fig 1c, baby lotion (EK) did not significantly improve barrier function both 1 and 5 hours after single application. However, Vaseline $^{\mathrm{TM}}$ (E) significantly improved TEWL 1 hour after a single application, but the benefits of Vaseline ${ }^{\mathrm{TM}}$ were not sustained at 5 hours after application.

\section{Influences of Topical Skin Care Products on Skin Surface pH and Stratum Corneum}

\section{Hydration}

Previous studies from our group have demonstrated that both the formation and the maintenance of a competent permeability barrier require an acidic $\mathrm{SC} \mathrm{pH} \mathrm{[42-44].} \mathrm{Since} \mathrm{most} \mathrm{of} \mathrm{the} \mathrm{products}$ tested here compromised basal permeability barrier function and barrier repair kinetics, we next assessed whether these products also change skin surface $\mathrm{pH}$. Indeed, all of products significantly elevated skin surface $\mathrm{pH}$ following 3 days of topical applications, with the exception of baby lotion (EK) and olive oil (EK) (Fig 2a). 
Because most products increase basal TEWL (Fig 1a), and changes in TEWL correlate with SC hydration [13], we next measured SC hydration following 3 days of treatment. Vaseline $\frac{\mathrm{TM}}{(\mathrm{E})}$ did not change SC hydration (Fig 2b), but both baby lotion (B) and baby oil (EK) elevated SC hydration, while the other tested products lowered SC hydration (Fig 2b).

\section{Discussion}

Previous studies have shown that improving the epidermal permeability barrier by topical emollients can reduce neonatal mortality rates in preterm infants $[45,46]$. Whether commonly used, locally available and inexpensive skin care products improve permeability barrier is barely known, particularly in Africa where the neonatal mortality rates are high [10]. In the present study, we show that the majority of the products commonly used in four study sites do not improve the permeability barrier in mice; instead, they compromise the barrier function in intact skin. The products that were particularly harmful were cow butter $(E)$, groundnut oil, neem oil (B), coconut oil and baby oil (T). The results from these mouse studies suggest that most currently used emollients could conceivably have a-harm the cutaneous permeability barrier of newborn humans; and consequently, they could contribute to neonatal mortality because of the central role of the permeability barrier in preventing infections. Previous studies have shown that perturbed permeability barrier not only facilitates microbial invasion $[22,47,48]$, a major cause for neonatal death $[48,49]$, but also predisposes to the development of dermatitis $[48,50]$. Skin surface $\mathrm{pH}$ is already higher than normal in neonates $[13,42,51]$, and the negative impact of these products on SC pH could not only further compromise the permeability barrier [44,52], but also favor microbial infections [22,24]. The potential negative impact of these products may be exacerbated in some settings by the way that these products are applied, which has been found to be forceful [34]. Only baby lotion (EK) showed some benefit in the mouse models, as it 
increased SC hydration without altering skin surface $\mathrm{pH}$, TEWL or barrier recovery. Vaseline ${ }^{\mathrm{TM}}$ from Ethiopia (E) did not alter SC hydration or barrier recovery, but did increase basal TEWL and minimally increased skin surface $\mathrm{pH}$. However, it did benefit mice with defective permeability barriers, suggesting that Vaseline ${ }_{\text {TM }}^{\mathrm{TM}}$-could benefit preterm neonates with defective permeability barriers. However, the impact of Vaseline ${ }^{\mathrm{TM}}$ was short-term, which supports prior studies that have shown that Vaseline ${ }^{\mathrm{TM}}$ exerts only short-term benefits for barrier-damaged skin [40,41]. Hence, frequent application would be required for this product to provide maximum benefit.

It is worth noting that certain products diversely impact epidermal functions. For example, Baby oil (B) significantly increased SC hydration (beneficialt), while dramatically damaging other epidermal functions, including increased basal TEWL and skin surface $\mathrm{pH}$, and delayed barrier recovery. Olive oil (EK) did not significantly alter basal TEWL or skin surface $\mathrm{pH}$, but induced a dramatic reduction in SC hydration and barrier recovery rate. Likewise, baby lotion (EK) significantly increased SC hydration without provoking significant alterations in basal TEWL, barrier recovery rate and skin surface $\mathrm{pH}$. Although baby lotion (EK) benefits SC hydration while Vaseline $^{\mathrm{TM}}$ (E) improves permeability barrier recovery, further studies would be required to determine whether a combination of these two products could further benefit skin with compromised epidermal function.

Nevertheless, given these findings, trials of alternative products that may enhance permeability barrier function in newbernsinfants, such as sunflower oil, should be undertaken in Africa, particularly in preterm infants. Given that we found that the effects of similar skin care products on permeability barrier homeostasis varied with their sources, possibly because small differences in composition, processing and/or storage could result in functional differences after applications 
to the skin, any future trials should take careful note of the source, manufacturing details and storage of the emollients used.

This study had some limitations. Moreover, it is worth noting that adult Adult mice were used in the present study. The, and the results may therefore not exactly reflect the influences of products on newborn mice or humans. Hence, further studies would be required to assess whether they also are harmful to neonatal human epidermis aAlthough these results strongly suggest a likely negative impact of the tested products on newborn skin, further studies would be required to assess whether they also are harmful to neonatal human epidermis. Moreover, although the present study tested products used in certain African regions, the benefits of any neonatal skin care products should be also validated before being put-placed on the market in other countries.

The variation in impact by source may explain why previous studies have found that coconut oil benefits newborn skin [2], while our study found it harmful to mouse skin. In Nigeria, another explanation for the varied findings is that counterfeiting of products has been identified as a problem [34].

\section{Conclusions}

Certain skin care products, which are widely used in neonatal care in Africa, are harmful to the function of murine skin. Although previous studies have demonstrated that the impact of topical products on human skin is consistent with those on mice, whether these products negatively influence human epidermal function remains unknown. These results further suggest that most currently used emollients likely have a harmful impact on the permeability barrier of newborns, which could result in increased body fluid loss, impaired thermoregulation, while also predisposing to perinatal infections. Hence, use of these potentially harmful skin care products 
could explain, at least in part, the high rates of neonatal morbidity and mortality that occur in Africa. Accordingly, studies of alternative products that enhance permeability barrier function should be undertaken, particularly in preterm infants.

Acknowledgements: This work was supported in part by the resources and uses of facilities at the Veterans Affairs Medical Center, San Francisco, California, USA.These studies were supported by NIH grant, AR019098 and a grant from Bill and Melinda Gates Foundation.

\section{References}

1. http://data.worldbank.org/indicator/SH.DYN.NMRT?order=wbapi_data_value_2013+wbapi _data_value+wbapi_data_value-last\&sort=desc (obtained on July 22, 2015)

2. Liu L, Oza S, Hogan D, et al. Global, regional, and national causes of child mortality in 2000-13, with projections to inform post-2015 priorities: an updated systematic analysis. Lancet. 2015;385:430-40

3. Million Death Study Collaborators, et al. Causes of neonatal and child mortality in India: a nationally representative mortality survey. Lancet. 2010;376:1853-60.

4. Liu L, Johnson HL, Cousens S, et al. Global, regional, and national causes of child mortality: an updated systematic analysis for 2010 with time trends since 2000. Lancet. 2012;379:215161. 
5. http://www.who.int/mediacentre/factsheets/fs363/en/ (obtained on June 18, 2015)

6. MacDorman MF, Matthews TJ, Mohangoo AD, et al. International comparisons of infant mortality and related factors: United States and Europe, 2010. Natl Vital Stat Rep. 2014;63:1-6.

7. GBD 2013 Mortality and Causes of Death Collaborators. Global, regional, and national agesex specific all-cause and cause-specific mortality for 240 causes of death, 1990-2013: a systematic analysis for the Global Burden of Disease Study 2013. Lancet. 2015;385:117-71.

8. http://www.who.int/maternal_child_adolescent/topics/newborn/care_of preterm/en/ (obtained on January 4, 2016)

9. Liu L, Oza S, Hogan D, et al. Global, regional, and national causes of child mortality in 2000-13, with projections to inform post-2015 priorities: an updated systematic analysis. Lancet. 2015;385:430-40.

10. http://data.worldbank.org/indicator/SH.DYN.NMRT?order=wbapi_data_value_2013+wbapi data_value+wbapi_data_value-last\&sort=desc (obtained on June 18, 2015)

11. Bahl R, Martines J, Ali N, et al. Research priorities to reduce global mortality from newborn infections by 2015. Pediatr Infect Dis J. 2009;28:S43-8.

12. Walker VP, Akinbi HT, Meinzen-Derr J, et al. Host defense proteins on the surface of neonatal skin: implications for innate immunity. J Pediatr. 2008;152:777-81.

13. Yosipovitch G, Maayan-Metzger A, Merlob P, et al. Skin barrier properties in different body areas in neonates. Pediatrics. 2000;106:105-8.

14. Nikolovski J, Stamatas GN, Kollias N, et al. Barrier function and water-holding and transport properties of infant stratum corneum are different from adult and continue to develop through the first year of life. J Invest Dermatol. 2008;128:1728-36. 
15. Hammarlund K, Sedin G. Water evaporation and heat exchange with the environment in newborn infants. Acta Paediatr Scand Suppl. 1983;305:32-5.

16. Hammarlund K, Sedin G, Strömberg B. Transepidermal water loss in newborn infants. VII. Relation to post-natal age in very pre-term and full-term appropriate for gestational age infants. Acta Paediatr Scand. 1982;71:369-74.

17. Kanti V, Bonzel A, Stroux A, et al. Postnatal maturation of skin barrier function in premature infants. Skin Pharmacol Physiol. 2014;27:234-41.

18. Hammarlund KM, Sedin G. Transepidermal water loss in newborn infants III. Relation to gestational age. Semin Neonatol 2000; 5:281-287.

19. Kalia YN, Nonato LB, Lund CH, et al. Development of skin barrier function in premature infants. J Invest Dermatol. 1998;111:320-6.

20. Agren J, Sjörs G, Sedin G. Ambient humidity influences the rate of skin barrier maturation in extremely preterm infants. J Pediatr. 2006; 148:613-7.

21. Hammarlund K, Strömberg B, Sedin G. Heat loss from the skin of preterm and fullterm newborn infants during the first weeks after birth. Biol Neonate. 1986;50:1-10.

22. Wanke I, Skabytska Y, Kraft B, et al. Staphylococcus aureus skin colonization is promoted by barrier disruption and leads to local inflammation. Exp Dermatol. 2013;22:153-5.

23. Conner JM, Soll RF, Edwards WH. Topical ointment for preventing infection in preterm infants. Cochrane Database Syst Rev. 2004;(1):CD001150.

24. Chiller K, Selkin BA, Murakawa GJ. Skin microflora and bacterial infections of the skin. J Investig Dermatol Symp Proc. 2001;6:170-4.

25. Sung SI, Ahn SY, Seo HJ, et al. Insensible Water Loss during the First Week of Life of Extremely Low Birth Weight Infants Less than 25 Gestational Weeks under High 
Humidification. Neonatal Med. 2013;20:51-57.

26. Maurer A, Micheli JL, Schütz Y, et al. Transepidermal water loss and resting energy expenditure in preterm infants. Helv Paediatr Acta. 1984;39:405-18.

27. Thijs H, Massawe AW, Okken A, Measurement of transepidermal water loss in Tanzanian cot-nursed neonates and its relation to postnatal weight loss. Acta Paediatr. 1996;85:356-60.

28. Salam RA, Das JK, Darmstadt GL, et al. Emollient therapy for preterm newborn infants-evidence from the developing world. BMC Public Health 2013;13 Suppl 3:S31.

29. Darmstadt GL, Ahmed S, Ahmed AN, et al. Mechanism for Prevention of Infection In Preterm Neonates by Topical Emollients: A Randomized, Controlled Clinical Trial. Pediatr Infect Dis J 2014; 33:1124-7.

30. Darmstadt GL, Saha SK, Ahmed AS, et al. Effect of topical emollient treatment of preterm neonates in Bangladesh on invasion of pathogens into the bloodstream. Pediatr Res. 2007;61:588-93.

31. Darmstadt GL, Saha SK, Ahmed AS, et al. Effect of topical treatment with skin barrierenhancing emollients on nosocomial infections in preterm infants in Bangladesh: a randomised controlled trial. Lancet. 2005;365:1039-45.

32. Darmstadt GL, Badrawi N, Law PA, et al. Topically applied sunflower seed oil prevents invasive bacterial infections in preterm infants in Egypt: a randomized, controlled clinical trial. Pediatr Infect Dis J. 2004;23:719-25.

33. Darmstadt GL, Mao-Qiang M, Chi E, et al. Impact of topical oils on the skin barrier: possible implications for neonatal health in developing countries. Acta Paediatr 2002;91:546-54.

34. Amare Y, Shamba D, Manzi F, et al. Current neonatal skin care practices in four African sites. Journal Topical Pediatrics. 2015; 61:428-34. 
35. Gudjonsson JE, Johnston A, Dyson M, et al. Mouse models of psoriasis.J Invest Dermatol. 2007; 127:1292-308.

36. Evans NJ, Rutter N. Development of the epidermis in the newborn. Biol Neonate. 1986;49:74-80.

37. Zettersten EM, Ghadially R, Feingold KR, et al. Optimal ratios of topical stratum corneum lipids improve barrier recovery in chronologically aged skin. J Am Acad Dermatol. $1997 ; 37: 403-8$

38. Man MQ, Barish GD, Schmuth M, et al. Deficiency of PPARbeta/delta in the epidermis results in defective cutaneous permeability barrier homeostasis and increased inflammation. $\mathrm{J}$ Invest Dermatol. 2008;128:370-7.

39. Man G, Cheung C, Crumrine D, et al. An Optimized Inexpensive Emollient Mixture Improves Barrier Repair in Murine Skin. Dermatologica Sinica. 2015; 33:96-102.

40. Ghadially R, Halkier-Sorensen L, Elias PM. Effects of petrolatum on stratum corneum structure and function. J Am Acad Dermatol. 1992;26:387-96.

41. Mao-Qiang M, Brown BE, Wu-Pong S, et al. Exogenous nonphysiologic vs physiologic lipids. Divergent mechanisms for correction of permeability barrier dysfunction. Arch Dermatol. 1995;131:809-16.

42. Behne MJ, Barry NP, Hanson KM, et al. Neonatal development of the stratum corneum $\mathrm{pH}$ gradient: localization and mechanisms leading to emergence of optimal barrier function. $\mathbf{J}$ Invest Dermatol. 2003;120:998-1006.

43. Fluhr JW, Mao-Qiang M, Brown BE, et al. Functional consequences of a neutral pH in neonatal rat stratum corneum. J Invest Dermatol. 2004;123:140-51. 
44. Mauro T, Holleran WM, Grayson S, et al. Barrier recovery is impeded at neutral pH, independent of ionic effects: implications for extracellular lipid processing. Arch Dermatol Res. 1998;290:215-22.

45. Darmstadt GL, Saha SK, Ahmed AS, et al. Effect of skin barrier therapy on neonatal mortality rates in preterm infants in Bangladesh: a randomized, controlled, clinical trial. Pediatrics. 2008;121:522-9.

46. Bhutta ZA, Das JK, Bahl R, et al. Can available interventions end preventable deaths in mothers, newborn babies, and stillbirths, and at what cost? Lancet. 2014;384:347-70.

47. Ray TL, Wuepper KD. Experimental cutaneous candidiasis in rodents; II. Role of the stratum corneum barrier and serum complement as a mediator of a protective inflammatory response.Arch Dermatol. 1978;114:539-43.

48. Jinnestål CL, Belfrage E, Bäck O, et al. Skin barrier impairment correlates with cutaneous Staphylococcus aureus colonization and sensitization to skin-associated microbial antigens in adult patients with atopic dermatitis. Int J Dermatol. 2014;53:27-33.

49. Jehan I, Harris H, Salat S, et al. Neonatal mortality, risk factors and causes: a prospective population-based cohort study in urban Pakistan. Bull World Health Organ. 2009;87:130-8.

50. Scharschmidt TC, Man MQ, Hatano Y, et al. Filaggrin deficiency confers a paracellular barrier abnormality that reduces inflammatory thresholds to irritants and haptens. J Allergy Clin Immunol. 2009;124:496-506

51. Fluhr JW, Behne MJ, Brown BE, et al. Stratum corneum acidification in neonatal skin: secretory phospholipase A2 and the sodium/hydrogen antiporter-1 acidify neonatal rat stratum corneum. J Invest Dermatol. 2004;122:320-9. 
52. Hachem JP, Roelandt T, Schürer N, et al. Acute acidification of stratum corneum membrane domains using polyhydroxyl acids improves lipid processing and inhibits degradation of corneodesmosomes. J Invest Dermatol. 2010;130:500-10.

\section{Figure Legends}

Figure 1. Effects of Topical Skin Care Products on Epidermal Permeability Barrier. Mice were treated as described in the materials and method section. Basal epidermal function was measured 18 hours after last application. Fig 1A depicts the influences of topical skin care products on basal transepidermal water loss (TEWL) after 3 days of treatment $(\mathrm{N}=20)$. TEWL was measured 16 hours after the last application. Fig 1B displays the effects of topical skin care products on permeability barrier homeostasis 3 hours after barrier disruption with repeated tape stripping $(\mathrm{N}=20)$. And-Fig 1C shows the effects of topical skin care products on permeability barrier homeostasis 1 and 5 hours after a single application to barrier disrupted murine skin

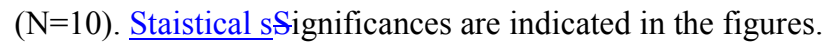


Figure 2. Effects of Topical Skin Care Products on Skin Surface pH and Stratum Corneum

Hydration. After 3 days of treatments with respective skin care product, stratum corneum hydration and skin surface $\mathrm{pH}$ were measured with a CM825 probe connected to MPA5 (C\&K,

Cologne, Germany). pH900 (C\&K, Cologne, Germany) was used to measure skin surface pH.

Results were normalized by untreated controls as shown by dotted lines. Statistical

$\underline{\text { s} S i g n i f i c a n c e s}$ are indicated in the figures. $\mathrm{N}=20$ for all. 